\title{
Comparative Performance of mmWave 5G System for many Beamforming Methods
}

\author{
Shaymaa A. Ahmed ${ }^{1}$, Saad A. Ayoob ${ }^{2}$, Ali Othman Al Janaby ${ }^{3}$ \\ \{ shymaa.enp9@student.uomosul.edu.iq ${ }^{1}$, Sa_ah_ay@uomosul.edu.iq ${ }^{2}$, \\ ali.mohammed@uoninevah.edu.iq ${ }^{3}$ \} \\ The University of Al Mosul ${ }^{1,2}$ \\ Ninevah University ${ }^{3}$
}

\begin{abstract}
With the massive propagation of the mobile phone traffic and demand for high data rate transmission has grown this day, the current cellular spectrum for bands less than $3 \mathrm{GHz}$ is severely deficient and cannot convoy these requirements, which requires the search for suitable solutions for the application of the fifth generation $5 \mathrm{G}$. The potential of millimetre-wave (mm-wave) to provide a high data rate (Gigabits per second) has attracted great interest to become a broadband candidate for the fifth generation of cellular communications networks. To increase the spectral efficiency of cellular networks, Massive multi-input multi output (MMIMO) is considered one of the most promising methods, by equipping the base station with antenna arrays with hundreds or thousands of active elements with the use of beamforming technology and conducting coherent processing on both sides of the transmitter and receiver. In this paper, two mm-wave frequencies $(28 \mathrm{GHz}$ and $73 \mathrm{GHz})$ have been compared in terms of spectrum efficiency using a massive MIMO using two beamforming methods: conjugate beamforming $(\mathrm{CB})$ and zero-forcing $(\mathrm{ZF})$.
\end{abstract}

Keywords: Beamforming, Massive MMIMO, mm-wave, CB, ZF, 5G.

\section{Introduction}

Increasing demands from users for an advanced communication mode, in addition to voice communication, have led to significant growth in the volume of data traffic with the increased use of video communications, social media communication, and access to cloud data. To meet these requirements, it was necessary to use higher frequencies than in the fourth generation, and thus use more bandwidth. The frequency range $(30-300 \mathrm{GHz})$ has been receiving a lot of interest in the fifth-generation communications in recent times, as it was called the millimetre wave (mm Wave) because it has a short wavelength measured in millimetres. To meet the requirements of the fifth generation, the main key to success in achieving the required large data rates is expected to be using millimetre-wave in communications. But the main problem with the use of millimetre-wave is its short propagation distance, which leads to signal attenuation due to oxygen absorption, object blocking, and signal scattering problems as this can lead to the limited coverage of the eNodeB base station. To alleviate this problem, it was necessary to use a massive MIMO technology [1][2]. MIMO technology uses multiple antennas in transceivers to transfer more data simultaneously. Attenuation due to millimetre-wave weakening cannot be compensated with MIMO technology. Thus, more data rate can be provided in addition to more 
power gain to compensate for millimetre-wave attenuation by adopting Massive multi-input multi-output (MMIMO)instead of MIMO technology [3].

The main contribution of this paper can be explained as follows:

1. We compute the spectral efficiency to the number of UEs $\mathrm{k}$ by proposing downlink MU massive MIMO on mm-wave channel with the two precoding schemes ZF, $\mathrm{CB}$ at indoor LOS environments by using two frequencies $(28 \mathrm{GHz}$ and $73 \mathrm{GHz})$.

2. We increase the number of BS antennas from 100 to 500 and show how this influences spectral efficiency.

3. Our results show that the spectral efficiency increases by increasing the number of BS antennas (N) for different linear precoding schemes. We also concluded that a better spectral efficiency can be obtained at $28 \mathrm{GHz}$.

The other paragraphs of this paper will be prepared as follows; Section 2 reviews the related work. Section 3 overviews the 5G. Section 4 presents the proposed scheme. Finally, section 5 completes the simulation evaluation and conclusion.

\section{Related works}

In [4], the author presents a 3D statistical channel model for millimetre-wave communications LOS and NLOS for the link, local area, and tracking CIRs, with random carrier frequency, signal bandwidth, and antenna bandwidth, which is invaluable in the design of the fifth generation of cellular millimetre-wave networks. and random antenna frequency, they show good agreement between the model and the published RMS DS as well as a MATLABbased statistical simulator was implemented to generate a large set of PDPs and 3D power angle spectra, which shows good agreement with field measurements, thus validating 3-D SSCM to design the next generation of wireless systems that will use sub- $6 \mathrm{GHz}$ and $\mathrm{mm}$ Wave models to design filters, multi-element antenna arrays, and mm Wave transceivers.

In [5], the researchers compared three large-scale propagation path loss candidate models for use over the entire microwave and millimetre-wave radio spectrum: the mm Wave alpha-betagamma (ABG) model, the near free space reference distance (CI) model, and the $\mathrm{CI}$ model with the exponent of frequency-weighted path loss (CIF).

In [6], the authors used a downlink multi-user $\mathrm{mm}$ Wave system with an efficient hybrid analogue/digital beamforming algorithm for systems. and demonstrated that massive multiuser MIMO could be an ideal candidate for a mm Wave communications system. And they proved that the proposed algorithm, through the simulation results, and with the increase in the number of users outperform the traditional hybrid beam configuration as it improved the sum rate and thus led to better performance for the bit error rate (BER).

In [7], the researchers presented specifics and applications of a new channel simulation software called NYUSIM, which can be used to configure actual temporal and spatial channel responses to uphold realistic physical layer simulations, link layer, and $5 \mathrm{G}$ cellular communication layout. In [8], The researchers describe the results of an internal experimental validation test and discuss a multi-user $28 \mathrm{GHz}$ multi-input (MIMO) output using of Active Digital MIMO System (AAS) 
(360 elements) for application on a 5G base station. This report was the first experimental validation of a multi-user MIMO using $28 \mathrm{GHz}$ digital AAS.

In [9], the researchers studied resource allocation as the need to increase capacity requires adding more antennas and the use of Massive multi-input multi-output (MMIMO) allows multiple users to be scheduled to use the same frequency resource simultaneously.

In [10], the researchers provided a comprehensive overview of the factors affecting millimetrewave propagation such as the effects of the surrounding environment (atmospheric attenuation, free space path loss, rain and foliage attenuation, material penetration losses) In addition, many factors influence the propagation properties of the wave. The researchers discussed the radiofrequency spectrum, organizing $5 \mathrm{G}$ issues, and the methods of modelling for the channel. Current channel scaling campaigns and modelling methods for the most interesting 5G communication scenarios, such as millimetre-wave communications, massive multi-input communications, multiple access, and performance analysis were also presented

In [11], this thesis is the first to introduce deep learning (DL) frame for channel estimation in a large smart surface (LIS) with the help of a multi-input system (MIMO). Explain his superior performance.

In [12], the researchers proposed power distribution in the small cell to reduce path loss by specifying large power and controlling the distributed power to reduce the high cost and power consumption where millimetre wave hybrid beamforming exploits large bandwidth which reduces the large path loss in the Rayleigh fading channel.

In [13], this paper, the researchers propose an intelligent downlink reflective surface (IRS) supported by non-orthogonal mm Wave multiple access (NOMA) and MIMO Massive antenna with lens antenna array where individual antenna users are grouped without direct correlation but are connected to the base station (BS) with the help of the IRS as a single NOMA group. The performance of the millimetre-wave beam area-weighted sum average supported by nonorthogonal multiple access (NOMA) was verified by simulations.

\section{An Overview of the Most Important 5G Technologies}

In this section, an overview of the $5 \mathrm{G}$ system massive multi-input multi output (MMIMO) and Millimetre Wave and Beamforming schemes:

\subsection{Massive MIMO Technique}

Massive Multi-Input Multi Output (MMIMO) technology is now a cornerstone of upcoming 5G systems, where base stations are equipped with hundreds or a few thousand arrays of antennas that achieves a lot of performance gains in terms of reliability, security, and improved efficiency (spectrum and energy) relative to traditional MIMO [14]. One of the most important ways to increase the efficiency of the spectrum is by sending many simultaneous parallel focus beams to serve each user individually in place of using a sole omnidirectional beam for serving all users, this can be achieved by using Massive Multi-Input Multi Output (MMIMO) as shown in Fig 1 [15]. Massive Multi-Input Multi-Output (MMIMO) use is cheaper than conventional systems by replacing 50-watt super linear amplifiers with hundreds of mill watt low power amplifiers[15][16]. Massive Multi-Input Multi Output (MMIMO) provides high energy efficiency as BS concentrates the emitted power on exactly spatial directions where the user is located and thus Massive Multi-Input Multi Output (MMIMO) reduces limitations on the 
linearity, accuracy, and RF gain requests of the amplifiers used [17]. It also reduces interference with other channels because it uses a tapered beam. The Massive multi-input multi-output (MMIMO) technology significantly reduces latency at the air interface because it uses many antennas and uses beamforming that solves the fading problem of the transmitted signal [15].

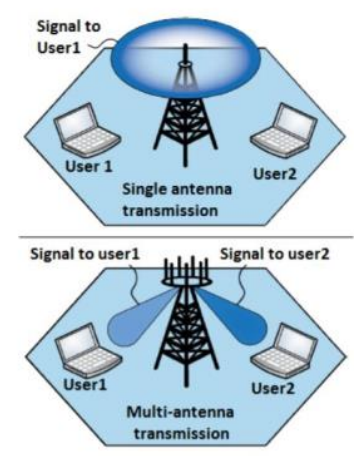

Fig. 1. Omnidirectional antenna and multi-directional antenna.

\subsection{Millimetre wave}

Millimetre-wave is one of the promising solutions to meet the unprecedented demands to provide a high data rate that keeps pace with the requirements of the fifth generation, due to its wide bandwidth [18]. The mm-wave contains frequency bands ranging from $(30-300) \mathrm{GHz}$ with wavelengths ranging from 1 to $100 \mathrm{~mm}$ and it has a basic 10Gb/s bandwidth access with the highest data rates [19][20]. However, due to its short wavelength, it suffers from large-signal attenuation caused by oxygen intake, object blocking, and signal scattering problems which lead to shortening of the wave propagation distance and thus shortening the coverage range of the eNodeB base station in mobile communications. Therefore, the use of Massive Multi-Input Multi-Output (MMIMO) and beamforming techniques is necessary to avoid the problems associated with using $\mathrm{mm}$ Wave [20].

\subsection{Beamforming}

The Massive Multi-Input Multi-Output (MMIMO) and mm Wave technologies provide solutions to many technical challenges facing the application $5 \mathrm{G}$ in the future. Spectrum and energy efficiency are enhanced in wireless communication networks by deploying a massive number of antennas in the transmitter and/or receiver (Massive Multi-Input Multi-Output (MMIMO)). To achieve the required performance gains in the environment in which the signal suffers from scattering, simple beamforming strategies must be used, such as the Transmission of the Maximum Rate (MRT) or Zero Forcing (ZF) [21]. Beamforming is a technique that focuses the signal on a beam in a specific direction towards a specific user rather than sending it equally in all directions. To increase the capacity, the beamforming technique must be applied, which leads to an increase in the strength of the received signal for each user while reducing the 
strength of the interference signal from other users [22]. ZF precoding considers the interference between users and disregards the effect of noise, while in MRT, BS is trying to maximize SNR received regardless of the effect of multi-user interference(5). We use two conventional linear detectors MRC and ZF and they can be modelled as shown in equation (1) [16]:

$\mathrm{A}= \begin{cases}G & \text { for } C B \\ G\left(G^{H} G\right)^{-1} & \text { for } Z F\end{cases}$

Where $\mathrm{A}$ is an $(\mathrm{N} \times \mathrm{K})$ linear detector matrix that depends on channel $\mathrm{G}$.

$\mathrm{H} \in C^{\mathrm{Nt} \times \mathrm{K}}$

$\mathrm{N}$ is the number of antennas and $\mathrm{K}$ is the number of UEs [16].

\section{Proposed Model}

As we mentioned earlier, a millimetre-wave is one of the most important solutions to add a huge amount of bandwidth that is currently required. But there are important components of its modelling, most notably [18]:

1- Spatial Consistency: Shows incessant and actual channel progress accompanying the user's equipment path within the local zone [23] [24].

2- Human Blockage Shadowing Loss: this means loss due to somebody close to the mobile phone who may block the transmission signal. This loss may cause several hundreds of milliseconds [25].

3- Outdoor-to-Indoor (O2I) penetration: modern buildings are fabricated by material that induced a big permeation loss for mm-wave signal transferred from outdoor to indoor and in opposite direction [26]. The wave channel model components are shown in Fig 2:

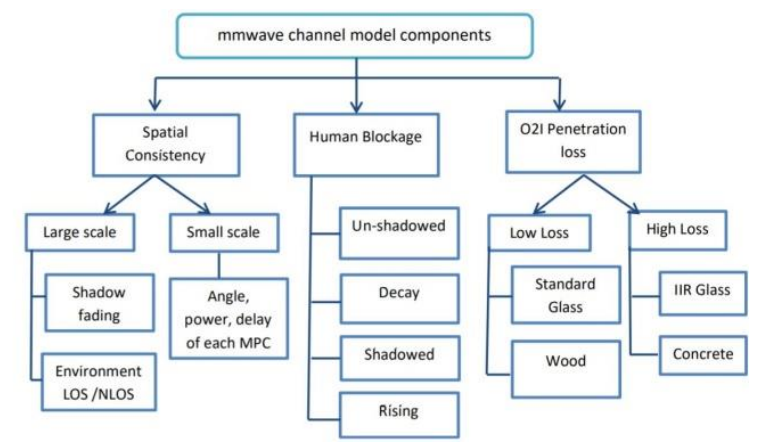

Fig. 2. mm-wave channel model components.

In addition to many important factors that go into calculating millimetre-wave path losses, such as barometric pressure, humidity, temperature, rain rate, polarization, and foliage loss [27]. We Propose a downlink multiuser Massive Multi-Input Multi-Output (MMIMO) with mm-wave 
channel by using two precoding schemes ZF, MRT which is also called conjugate beamforming (CB). Perfect channel estimation and small-scale fading channels are assumed. The LOS MIMO channel matrix, G, is modelled as shown in equation (2):

$$
G_{l}=\frac{\lambda}{4 \pi R} \exp \left(-j \frac{2 \pi}{\lambda} d_{l}\right)
$$

Which contains the $l_{t h}$ column of the $\mathrm{Nr} \times \mathrm{Nt}$.

Where $G_{l}$ indicates the $l_{\text {th }}$ transmit antenna vector which contains all channel routes between the $l$ antenna and all receive antennas, and $\lambda=\mathrm{c} / \mathrm{f}$, where $\lambda$ is carrier wavelength, and $\mathrm{c}$ is the speed of light, and $\mathrm{f}$ is the carrier frequency, and $\mathrm{R}$ represents the space between the transmitter antenna array and the receiver antenna array, and $d_{l}$ is the distance vector between $l$ transmit antenna and all receive antennas [28].

\section{Proposed Model Configuration and Simulation}

We study the performance of multi-user Massive multi-input multi output (MMIMO) mm Wave channels for the indoor environment by using MATLAB (m.file) and sketch spectral efficiency (SE) results concerning no. of UEs (K) by applying two beamforming methods, for example., Conjugate Beamforming (CB), Zero-Forcing (ZF) by using the assumptions in the Table 1.

Table 1. Simulation assumption.

\begin{tabular}{|c|c|}
\hline Parameter & Setting \\
\hline$d_{l}$ & $0.5 \lambda$ \\
\hline Transmit power & $30 \mathrm{dBm}$ \\
\hline $\begin{array}{l}\text { Carrier frequencies } \\
\text { No.of base station } \\
\text { antenna }(\mathrm{N})\end{array}$ & $\begin{array}{l}28 \mathrm{GHz}, 73 \mathrm{GHz} \\
100,500\end{array}$ \\
\hline $\begin{array}{l}\text { Noise variance } \\
\text { R } \\
\text { Path loss exponent } \\
\text { for } 28 \mathrm{GHz} \\
\text { Path loss exponent } \\
\text { for } 73 \mathrm{GHz}\end{array}$ & $\begin{array}{l}-80 \mathrm{dBm} \\
200 \mathrm{~m} \\
1.8-1.9 \\
2.0\end{array}$ \\
\hline
\end{tabular}

Fig 3 shows the comparison between the spectral efficiency versus no. of users (k) for $28 \mathrm{GHz}$ and $73 \mathrm{GHz}$ for indoor LOS environments at $\mathrm{N}=100$ and $\mathrm{N}=500$ respectively. 


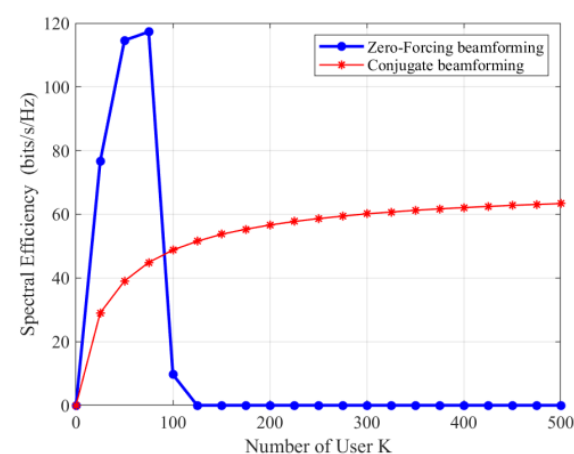

a $-\mathrm{F}=28 \mathrm{GHz}, \mathrm{N}=100$

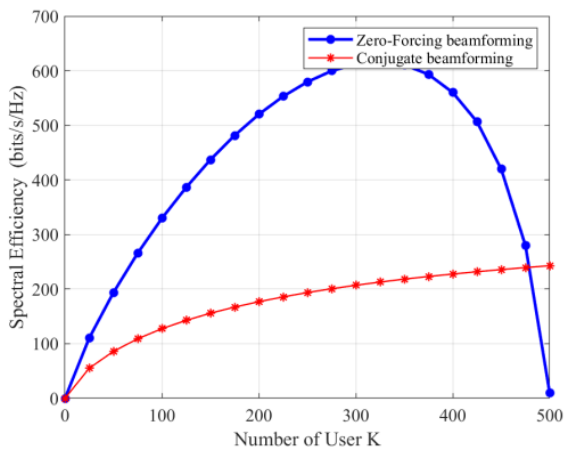

c- $\mathrm{F}=28 \mathrm{GHz}, \mathrm{N}=500$

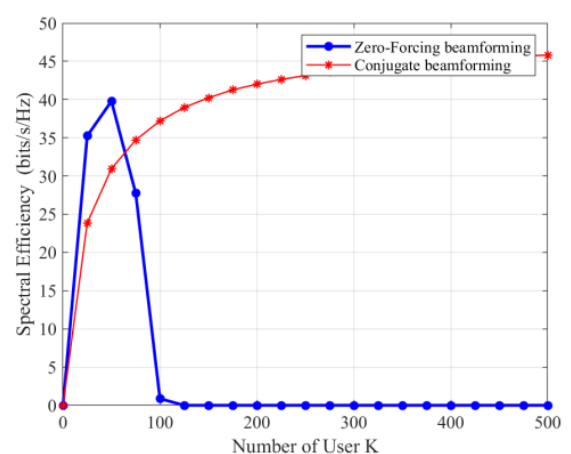

b-F=73GHz,N=100

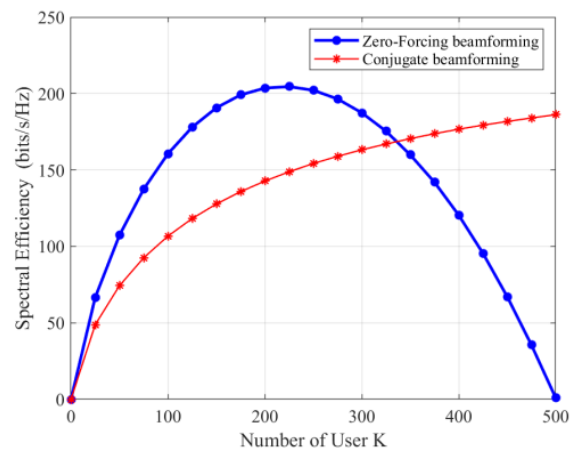

$\mathrm{d}-\mathrm{F}=73 \mathrm{GHz}, \mathrm{N}=500$

Fig. 3. Comparison of spectral efficiency for different frequencies $(28$ and $73 \mathrm{GHz})$ and different no. of antennas concerning no. of UEs $\mathrm{k}$.

We can summarize the above figures in the following Table 2:

Table 2. The maximum value of the spectrum efficiency for ZF precoding schemes.

\begin{tabular}{lll}
\hline Frequency $(\mathrm{GHz})$ & No.of BS antennas & $\begin{array}{l}\text { Spectral } \\
\text { efficiency }(\mathrm{bit} / \mathrm{sec} / \mathrm{Hz})\end{array}$ \\
\hline 28 & 100 & 117.2 \\
28 & 500 & 612.3 \\
73 & 100 & 39.79 \\
73 & 500 & 203.6 \\
\hline
\end{tabular}




\section{Conclusion}

Massive multi-input multi-output (MMIMO) with mm-wave is the most enabling key for the next generation of communication. In this paper, we compare two mm-wave frequencies; for example, $28 \mathrm{GHz}$ and $73 \mathrm{GHz}$ by applying multi- user Massive Multi-Input Multi-Output (MMIMO) and obtain the spectral efficiency results by using Zero-Forcing (ZF) and conjugate beamforming precoding schemes. The results show that the spectral efficiency at $28 \mathrm{GHz}$ is better than it at $73 \mathrm{GHz}$ and show that there is a remarkable improvement in the spectral efficiency when the no. of BS antennas increased. We also note that the ZF precoding scheme outperforms the $\mathrm{CB}$, but when no. of UEs increased we note that $\mathrm{CB}$ is better than $\mathrm{ZF}$ because its arithmetic burden scales as (MK) while $\mathrm{ZF}$ complexity scales as $\left(\mathrm{M} K^{2}\right)$.

\section{Acknowledgement}

This work was supported by the Electrical Department, Engineering College, Mosul University.

\section{References}

[1] Islam MS, Kamruzzaman M, Jessy T, Zahan MS, Hassan MS. Performance Analysis of Massive MIMO for 5G Wireless Communication Systems. International Conference on Computing, Communication, and Automation (ICCCA2016); 29-30 April 2016; Greater Noida, India. IEEE; 16 January 2017. p. 1579-1583.

[2] Poudel KN, Gangaju S. Spectral Efficiency, Diversity Gain, Multiplexing, Capacity Analysis for Massive MIMO, 5G Communication System. International Conference on Networking and Network Applications; 16-19 Oct. 2017; Kathmandu, Nepal. IEEE; 08 January 2018. p. 133-137.

[3] Phuangkor W, Uthansakul P. Gain Compensation of Massive MIMO for Shifting from 4G to 5G Mobile Communications. $201911^{\text {th }}$ International Conference on Information Technology and Electrical Engineering (ICITEE) ; 10-11 Oct. 2019 ; Pattaya, Thailand. IEEE; 12 December 2019. p. $1-6$.

[4] Samimi MK, Rappaport TS. 3-D millimeter-wave statistical channel model for 5G wireless system design. IEEE Transactions on Microwave Theory and Techniques. 2016 Jun 28;64(7):2207-25.

[5] Sun S, Rappaport TS, Thomas TA, Ghosh A, Nguyen HC, Kovács IZ, Rodriguez I, Koymen O, Partyka A. Investigation of prediction accuracy, sensitivity, and parameter stability of large-scale propagation path loss models for $5 \mathrm{G}$ wireless communications. IEEE Transactions on Vehicular Technology. 2016 Mar 16;65(5):2843-60.

[6] Jing J, Xiaoxue C, Yongbin X. Energy-efficiency based downlink multi-user hybrid beamforming for millimeter wave massive MIMO system. The Journal of China Universities of Posts and Telecommunications. 2016 Aug 1;23(4):53-62.

[7] Sun S, MacCartney GR, Rappaport TS. A novel millimeter-wave channel simulator and applications for 5G wireless communications. In2017 IEEE International Conference on Communications (ICC) ;2017 May 21 ; Paris, France. IEEE; 31 July 2017. p. 1-7).

[8] Manini M, Gueguen C, Legouable R, Lagrange X. Study of MIMO channel matrices correlation to optimize resource allocation algorithms in multi-users 5G. In2019 12th IFIP Wireless and Mobile Networking Conference (WMNC) ; 11-13 Sept. 2019; Paris, France. IEEE; 24 October 2019. p. 162166 .

[9] Uwaechia AN, Mahyuddin NM. A comprehensive survey on millimeter wave communications for fifth-generation wireless networks: feasibility and challenges. IEEE Access. 2020 Mar 30;8:62367-414. 
[10] Elbir AM, Papazafeiropoulos A, Kourtessis P, Chatzinotas S. Deep channel learning for large intelligent surfaces aided mm-wave massive MIMO systems. IEEE Wireless Communications Letters. 2020 May 11;9(9):1447-51.

[11] Abdullah Q, Salh A, MohdShah NS, Abdullah N, Audah L, Hamzah SA, Farah N, Aboali M, Nordin S. a Brief Survey and Investigation of Hybrid Beamforming for Millimeter Waves In 5G Massive MIMO Systems. arXiv preprint arXiv:2105.00180. 2021 May 1

[12] Liu P, Li Y, Cheng W, Gao X, Huang X. Intelligent Reflecting Surface Aided NOMA for MillimeterWave Massive MIMO with Lens Antenna Array. IEEE Transactions on Vehicular Technology. 2021 Mar 23.

[13] Ju S, Xing Y, Kanhere O, Rappaport TS. Millimeter wave and sub-terahertz spatial statistical channel model for an indoor office building. IEEE Journal on Selected Areas in Communications. $2021 \mathrm{Apr}$ 23;39(6):1561-75.

[14] Larsson EG, Edfors O, Tufvesson F, Marzetta TL. Massive MIMO for next generation wireless systems. IEEE communications magazine. 2014 Feb 12;52(2):186-95.

[15] A.O.M. Al Janaby, "5G downlink throughput enhancement by beam consolidation at vacant traffic," Journal of Communications Software and Systems, vol. 15, no. 4, pp. 311-316, Dec. 2019.

[16] Ngo HQ, Larsson EG, Marzetta TL. Energy and spectral efficiency of very large multiuser MIMO systems. IEEE Transactions on Communications. 2013 Feb 7;61(4):1436-49.

[17] Rusek F, Persson D, Lau BK, Larsson EG, Marzetta TL, Edfors O, Tufvesson F. Scaling up MIMO: Opportunities and challenges with very large arrays. IEEE signal processing magazine. 2012 Dec 10;30(1):40-60.

[18] Ju S, Kanhere O, Xing Y, Rappaport TS. A millimeter-wave channel simulator NYUSIM with spatial consistency and human blockage. In2019 IEEE Global Communications Conference (GLOBECOM) ; 9-13 Dec. 2019 ; Waikoloa, HI, USA. IEEE ; 27 February 2020 . p. 1-6.

[19] Budalal AA, Islam MR, Habaebi MH, Rahman TA. Millimeter Wave Channel Modeling-Present Development and Challenges in Tropical Areas. In2018 7th International Conference on Computer and Communication Engineering (ICCCE); 2018 Sep 19; Kuala Lumpur, Malaysia . IEEE; 19 November 2018. p. 23-28.

[20] Bogale TE, Le LB. Massive MIMO and mmWave for 5G wireless HetNet: Potential benefits and challenges. IEEE Vehicular Technology Magazine. 2016 Feb 3;11(1):64-75.

[21] Swindlehurst AL, Ayanoglu E, Heydari P, Capolino F. Millimeter-wave massive MIMO: The next wireless revolution. IEEE Communications Magazine. 2014 Sep;52(9):56-62.

[22] Ali E, Ismail M, Nordin R, Abdulah NF. Beamforming techniques for massive MIMO systems in 5G: overview, classification, and trends for future research. Frontiers of Information Technology \& Electronic Engineering. 2017 Jun;18(6):753-72.

[23] Ju S, Rappaport TS. Simulating motion-incorporating spatial consistency into nyusim channel model. In2018 IEEE 88th Vehicular Technology Conference (VTC-Fall) ;2018 Aug 27 ; Chicago, IL, USA . IEEE; 15 April 2019 . p. 1-6.

[24] Ju S, Rappaport TS. Millimeter-wave extended NYUSIM channel model for spatial consistency. In2018 IEEE Global Communications Conference (GLOBECOM); 2018 Dec 9; Abu Dhabi, United Arab Emirates. IEEE; 21 February 2019 .p. 1-6.

[25] MacCartney GR, Rappaport TS, Rangan S. Rapid fading due to human blockage in pedestrian crowds at 5G millimeter-wave frequencies. InGLOBECOM 2017-2017 IEEE Global Communications Conference; 2017 Dec 4; Singapore.IEEE; 15 January 2018. p. 1-7.

[26] Haneda K, Zhang J, Tan L, Liu G, Zheng Y, Asplund H, Li J, Wang Y, Steer D, Li C, Balercia T. 5G 3GPP-like channel models for outdoor urban microcellular and macrocellular environments. In2016 IEEE 83rd vehicular technology conference (VTC spring);2016 May 15; Nanjing, China .IEEE; 07 July 2016 .p. 1-7.

[27] Liebe HJ, Hufford GA, Cotton MG. Propagation modeling of moist air and suspended water/ice particles at frequencies below $1000 \mathrm{GHz}$. In AGARD. 1993 Nov.

[28] Raed M, Abdelhamid A. Space modulation techniques. New Jersey, USA: Wiley; 2018 May 8.chapter 7, SMTs for Millimeter-Wave Communications; p. 171-183 